\title{
Distance domination and distance irredundance in graphs
}

\author{
Adriana Hansberg, Dirk Meierling and Lutz Volkmann \\ Lehrstuhl II für Mathematik, RWTH Aachen University, 52056 Aachen, Germany \\ e-mail: \{hansberg,meierling,volkm\}@math2.rwth-aachen.de
}

Submitted: Feb 13, 2007; Accepted: Apr 25, 2007; Published: May 9, 2007

Mathematics Subject Classification: 05C69

\begin{abstract}
A set $D \subseteq V$ of vertices is said to be a (connected) distance $k$-dominating set of $G$ if the distance between each vertex $u \in V-D$ and $D$ is at most $k$ (and $D$ induces a connected graph in $G$ ). The minimum cardinality of a (connected) distance $k$-dominating set in $G$ is the (connected) distance $k$-domination number of $G$, denoted by $\gamma_{k}(G)\left(\gamma_{k}^{c}(G)\right.$, respectively). The set $D$ is defined to be a total $k$-dominating set of $G$ if every vertex in $V$ is within distance $k$ from some vertex of $D$ other than itself. The minimum cardinality among all total $k$-dominating sets of $G$ is called the total $k$-domination number of $G$ and is denoted by $\gamma_{k}^{t}(G)$. For $x \in X \subseteq V$, if $N^{k}[x]-N^{k}[X-x] \neq \emptyset$, the vertex $x$ is said to be $k$-irredundant in $X$. A set $X$ containing only $k$-irredundant vertices is called $k$-irredundant. The $k$-irredundance number of $G$, denoted by $\operatorname{ir}_{k}(G)$, is the minimum cardinality taken over all maximal $k$-irredundant sets of vertices of $G$. In this paper we establish lower bounds for the distance $k$-irredundance number of graphs and trees. More precisely, we prove that $\frac{5 k+1}{2} i r_{k}(G) \geq \gamma_{k}^{c}(G)+2 k$ for each connected graph $G$ and $(2 k+1) i r_{k}(T) \geq \gamma_{k}^{c}(T)+2 k \geq|V|+2 k-k n_{1}(T)$ for each tree $T=(V, E)$ with $n_{1}(T)$ leaves. A class of examples shows that the latter bound is sharp. The second inequality generalizes a result of Meierling and Volkmann [9] and Cyman, Lemańska and Raczek [2] regarding $\gamma_{k}$ and the first generalizes a result of Favaron and Kratsch [4] regarding $i r_{1}$. Furthermore, we shall show that $\gamma_{k}^{c}(G) \leq \frac{3 k+1}{2} \gamma_{k}^{t}(G)-2 k$ for each connected graph $G$, thereby generalizing a result of Favaron and Kratsch [4] regarding $k=1$.
\end{abstract}

Keywords: domination, irredundance, distance domination number, total domination number, connected domination number, distance irredundance number, tree

2000 Mathematics Subject Classification: 05C69 


\section{Terminology and introduction}

In this paper we consider finite, undirected, simple and connected graphs $G=(V, E)$ with vertex set $V$ and edge set $E$. The number of vertices $|V|$ is called the order of $G$ and is denoted by $n(G)$. For two distinct vertices $u$ and $v$ the distance $d(u, v)$ between $u$ and $v$ is the length of a shortest path between $u$ and $v$. If $X$ and $Y$ are two disjoint subsets of $V$, then the distance between $X$ and $Y$ is defined as $d(X, Y)=\min \{d(x, y) \mid x \in X, y \in Y\}$. The open $k$-neighborhood $N^{k}(X)$ of a subset $X \subseteq V$ is the set of vertices in $V \backslash X$ of distance at most $k$ from $X$ and the closed $k$-neighborhood is defined by $N^{k}[X]=$ $N^{k}(X) \cup X$. If $X=\{v\}$ is a single vertex, then we denote the (closed) $k$-neighborhood of $v$ by $N^{k}(v)\left(N^{k}[v]\right.$, respectively). The (closed) 1-neighborhood of a vertex $v$ or a set $X$ of vertices is usually denoted by $N(v)$ or $N(X)$, respectively $(N[v]$ or $N[X]$, respectively). Now let $U$ be an arbitrary subset of $V$ and $u \in U$. We say that $v$ is a private $k$-neighbor of $u$ with respect to $U$ if $d(u, v) \leq k$ and $d\left(u^{\prime}, v\right)>k$ for all $u^{\prime} \in U-\{u\}$, that is $v \in N^{k}[u]-N^{k}[U-\{u\}]$. The private $k$-neighborhood of $u$ with respect to $U$ will be denoted by $P N^{k}[u, U]\left(P N^{k}[u]\right.$ if $\left.U=V\right)$.

For a vertex $v \in V$ we define the degree of $v$ as $d(v)=|N(v)|$. A vertex of degree one is called a leaf and the number of leaves of $G$ will be denoted by $n_{1}(G)$.

A set $D \subseteq V$ of vertices is said to be a (connected) distance $k$-dominating set of $G$ if the distance between each vertex $u \in V-D$ and $D$ is at most $k$ (and $D$ induces a connected graph in $G$ ). The minimum cardinality of a (connected) distance $k$-dominating set in $G$ is the (connected) distance $k$-domination number of $G$, denoted by $\gamma_{k}(G)\left(\gamma_{k}^{c}(G)\right.$, respectively). The distance 1-domination number $\gamma_{1}(G)$ is the usual domination number $\gamma(G)$. A set $D \subseteq V$ of vertices is defined to be a total $k$-dominating set of $G$ if every vertex in $V$ is within distance $k$ from some vertex of $D$ other than itself. The minimum cardinality among all total $k$-dominating sets of $G$ is called the total $k$-domination number of $G$ and is denoted by $\gamma_{k}^{t}(G)$. We note that the parameters $\gamma_{k}^{c}(G)$ and $\gamma_{k}^{t}(G)$ are only defined for connected graphs and for graphs without isolated vertices, respectively.

For $x \in X \subseteq V$, if $P N^{k}[x] \neq \emptyset$, the vertex $x$ is said to be $k$-irredundant in $X$. A set $X$ containing only $k$-irredundant vertices is called $k$-irredundant. The $k$-irredundance number of $G$, denoted by $\operatorname{ir}_{k}(G)$, is the minimum cardinality taken over all maximal $k$-irredundant sets of vertices of $G$.

In 1975, Meir and Moon [10] introduced the concept of a $k$-dominating set (called a ' $k$-covering' in [10]) in a graph, and established an upper bound for the $k$-domination number of a tree. More precisely, they proved that $\gamma_{k}(T) \leq|V(T)| /(k+1)$ for every tree $T$. This leads immediately to $\gamma_{k}(G) \leq|V(G)| /(k+1)$ for an arbitrary graph $G$. In 1991, Topp and Volkmann [11] gave a complete characterization of the class of graphs $G$ that fulfill the equality $\gamma_{k}(G)=|V(G)| /(k+1)$.

The concept of $k$-irredundance was introduced by Hattingh and Henning [5] in 1995. With $k=1$, the definition of an $k$-irredundant set coincides with the notion of an irredundant set, introduced by Cockayne, Hedetniemi and Miller [1] in 1978. Since then a lot of research has been done in this field and results have been presented by many authors (see $[5])$. 
In 1991, Henning, Oellermann and Swart [8] motivated the concept of total distance domination in graphs which finds applications in many situations and structures which give rise to graphs.

For a comprehensive treatment of domination in graphs, see the monographs by Haynes, Hedetniemi and Slater [6], [7].

In this paper we establish lower bounds for the distance $k$-irredundance number of graphs and trees. More precisely, we prove that $\frac{5 k+1}{2} i r_{k}(G) \geq \gamma_{k}^{c}(G)+2 k$ for each connected graph $G$ and $(2 k+1) i r_{k}(T) \geq \gamma_{k}(T)+2 k \geq|V|+2 k-k n_{1}(T)$ for each tree $T=(V, E)$ with $n_{1}(T)$ leaves. A class of examples shows that the latter bound is sharp. Since $\gamma_{k}(G) \geq i r_{k}(G)$ for each connected graph $G$, the latter generalizes a result of Meierling and Volkmann [9] and Cyman, Lemanska and Raczek [2] regarding $\gamma_{k}$ and the former generalizes a result of Favaron and Kratsch [4] regarding $i r_{1}$. In addition, we show that if $G$ is a connected graph, then $\gamma_{k}^{c}(G) \leq(2 k+1) \gamma_{k}(G)-2 k$ and $\gamma_{k}^{c}(G) \leq \frac{3 k-1}{2} \gamma_{k}^{t}(G)-2 k$ thereby generalizing results of Duchet and Meyniel [3] for $k=1$ and Favaron and Kratsch [4] for $k=1$, respectively.

\section{Results}

First we show the inequality $\gamma_{k}^{c} \leq(2 k+1) \gamma_{k}-2 k$ for connected graphs.

Theorem 2.1. If $G$ is a connected graph, then

$$
\gamma_{k}^{c}(G) \leq(2 k+1) \gamma_{k}(G)-2 k
$$

Proof. Let $G$ be a connected graph and let $D$ be a distance $k$-dominating set. Then $G[D]$ has at most $|D|$ components. Since $D$ is a distance $k$-dominating set, we can connect two of these components to one component by adding at most $2 k$ vertices to $D$. Hence, we can construct a connected $k$-dominating set $D^{\prime} \supseteq D$ in at most $|D|-1$ steps by adding at most $(|D|-1) 2 k$ vertices to $D$. Consequently,

$$
\gamma_{k}^{c}(G) \leq\left|D^{\prime}\right| \leq|D|+(|D|-1) 2 k=(2 k+1)|D|-2 k
$$

and if we choose $D$ such that $|D|=\gamma_{k}(G)$, the proof of this theorem is complete.

The results given below follow directly from Theorem 2.1.

Corollary 2.2 (Duchet \& Meyniel [3] 1982). If $G$ is a connected graph, then

$$
\gamma^{c}(G) \leq 3 \gamma(G)-2
$$

Corollary 2.3 (Meierling \& Volkmann [9] 2005; Cyman, Lemańska \& Raczek [2] 2006). If $T$ is a tree with $n_{1}$ leaves, then

$$
\gamma_{k}(T) \geq \frac{|V(T)|-k n_{1}+2 k}{2 k+1} .
$$


Proof. Since $\gamma_{k}^{c}(T) \geq|V(T)|-k n_{1}$ for each tree $T$, the proposition is immediate.

The following lemma is a preparatory result for Theorems 2.5 and 2.7.

Lemma 2.4. Let $G$ be a connected graph and let $I$ be a maximal $k$-irredundant set such that $\operatorname{ir}_{k}(G)=|I|$. If $I_{1}=\left\{v \in I \mid v \in P N^{k}[v]\right\}$ is the set of vertices that have no $k$-neighbor in $I$, then

$$
\gamma_{k}^{c}(G) \leq(2 k+1) i r_{k}(G)-2 k+(k-1) \frac{\left|I-I_{1}\right|}{2} .
$$

Proof. Let $G$ be a connected graph and let $I \subseteq V$ be a maximal $k$-irredundant set. Let

$$
I_{1}:=\left\{v \in I \mid v \in P N^{k}[v]\right\}
$$

be the set of vertices in $I$ that have no $k$-neighbors in $I$ and let

$$
I_{2}:=I-I_{1}
$$

be the complement of $I_{2}$ in $I$. For each vertex $v \in I_{2}$ let $u_{v} \in P N^{k}[v]$ be a $k$-neighbor of $v$ such that the distance between $v$ and $u_{v}$ is minimal and let

$$
B:=\left\{u_{v} \mid v \in I_{2}\right\}
$$

be the set of these $k$-neighbors. Note that $|B|=\left|I_{2}\right|$. If $w$ is a vertex such that $w \notin$ $N^{k}[I \cup B]$, then $I \cup\{w\}$ is a $k$-irredundant set of $G$ that strictly contains $I$, a contradiction. Hence $I \cup B$ is a $k$-dominating set of $G$.

Note that $G[I \cup B]$ has at most $|I \cup B|=\left|I_{1}\right|+2\left|I_{2}\right|$ components. From $I \cup B$ we shall construct a connected $k$-dominating set $D \supseteq I \cup B$ by adding at most

$$
\left|I_{2}\right|(k-1)+\left(\left|I_{1}\right|+\left\lfloor\frac{\left|I_{2}\right|}{2}\right\rfloor-1\right) 2 k+\left\lfloor\frac{\left|I_{2}\right|}{2}\right\rfloor(k-1)
$$

vertices to $I \cup B$.

We can connect each vertex $v \in I_{2}$ with its corresponding $k$-neighbor $u_{v} \in B$ by adding at most $k-1$ vertices to $I \cup B$.

Recall that each vertex $v \in I_{2}$ has a $k$-neighbor $w \neq v$ in $I_{2}$. Therefore we need to add at most $k-1$ vertices to $I \cup B$ to connect such a pair of vertices.

By combining the two observations above, we can construct a $k$-dominating set $D^{\prime} \supseteq$ $I \cup B$ from $I \cup B$ with at most $\left|I_{1}\right|+\left\lfloor\left|I_{2}\right| / 2\right\rfloor$ components by adding at most $(k-1)\left|I_{2}\right|+$ $(k-1)\left\lceil\left|I_{2}\right| / 2\right\rceil$ vertices to $I \cup B$. Since $D^{\prime}$ is a $k$-dominating set of $G$, these components can be joined to a connected $k$-dominating set $D$ by adding at most $\left(\left|I_{1}\right|+\left\lfloor\left|I_{2}\right| / 2\right\rfloor-1\right) 2 k$ vertices to $D^{\prime}$.

All in all we have shown that there exists a connected $k$-dominating set $D$ of $G$ such that

$$
\begin{aligned}
|D| & \leq\left|I_{1}\right|+2\left|I_{2}\right|+(k-1)\left|I_{2}\right|+(k-1)\left\lceil\frac{\left|I_{2}\right|}{2}\right\rceil+2 k\left(\left|I_{1}\right|+\left\lfloor\frac{\left|I_{2}\right|}{2}\right\rfloor-1\right) \\
& \leq(2 k+1)|I|-2 k+(k-1) \frac{\left|I_{2}\right|}{2} .
\end{aligned}
$$


Hence, if we choose the set $I$ such that $|I|=i r_{k}(G)$, the proof of this lemma is complete.

Since $\left|I_{2}\right| \leq|I|$ for each $k$-irredundant set $I$, we derive the following theorem.

Theorem 2.5. If $G$ is a connected graph, then

$$
\gamma_{k}^{c}(G) \leq \frac{5 k+1}{2} i r_{k}(G)-2 k
$$

The next result follows directly from Theorem 2.5.

Corollary 2.6 (Favaron \& Kratsch [4] 1991). If $G$ is a connected graph, then

$$
\gamma^{c}(G) \leq 3 \operatorname{ir}(G)-2
$$

For acyclic graphs Lemma 2.4 can be improved as follows.

Theorem 2.7. If $T$ is a tree, then

$$
\gamma_{k}^{c}(T) \leq(2 k+1) i r_{k}(T)-2 k .
$$

Proof. Let $T$ be a tree and let $I \subseteq V$ be a maximal $k$-irredundant set. Let

$$
I_{1}:=\left\{v \in I \mid v \in P N^{k}[v]\right\}
$$

be the set of vertices in $I$ that have no $k$-neighbors in $I$ and let

$$
I_{2}:=I-I_{1}
$$

be the complement of $I_{2}$ in $I$. For each vertex $v \in I_{2}$ let $u_{v} \in P N^{k}[v]$ be a $k$-neighbor of $v$ such that the distance between $v$ and $u_{v}$ is minimal and let

$$
B:=\left\{u_{v} \mid v \in I_{2}\right\}
$$

be the set of these $k$-neighbors. Note that $|B|=\left|I_{2}\right|$. If $w$ is a vertex such that $w \notin$ $N^{k}[I \cup B]$, then $I \cup\{w\}$ is a $k$-irredundant set of $G$ that strictly contains $I$, a contradiction. Hence $I \cup B$ is a $k$-dominating set of $G$.

Note that $T[I \cup B]$ has at most $|I \cup B|=\left|I_{1}\right|+2\left|I_{2}\right|$ components. From $I \cup B$ we shall construct a connected $k$-dominating set $D \supseteq I \cup B$ by adding at most

$$
(2 k-1)\left|I_{2}\right|+2 k\left(\left|I_{1}\right|-1\right)
$$

vertices to $I \cup B$. To do this we need the following definitions. For each vertex $v \in I_{2}$ let $P_{v}$ be the (unique) path between $v$ and $u_{v}$ and let $x_{v}$ be the predecessor of $u_{v}$ on $P_{v}$. Let $I_{2}=S \cup L_{1} \cup L_{2}$ be a partition of $I_{2}$ such that

$$
S=\left\{v \in I_{2} \mid d\left(v, u_{v}\right)=1\right\}
$$


is the set of vertices of $I_{2}$ that are connected by a 'short' path with $u_{v}$,

$$
L_{1}=\left\{v \in I_{2} \mid N^{k}\left(x_{v}\right) \cap I_{1} \neq \emptyset\right\}
$$

is the set of vertices of $I_{2}$ that are connected by a 'long' path with $u_{v}$ and the vertex $x_{v}$ has a $k$-neighbor in $I_{1}$ and

$$
L_{2}=I_{2}-\left(S \cup L_{1}\right)
$$

is the complement of $S \cup L_{1}$ in $I_{2}$. In addition, let $L=L_{1} \cup L_{2}$. We construct $D$ following the procedure given below.

Step 0: Set $\mathcal{I}:=I_{2}, \mathcal{S}:=S$ and $\mathcal{L}:=L$.

Step 1: We consider the vertices in $\mathcal{S}$.

Step 1.1: If there exists a vertex $v \in \mathcal{S}$ such that $d(v, w) \leq k$ for a vertex $w \in \mathcal{L}$, we can connect the vertices $v, u_{v}, w$ and $u_{w}$ to one component by adding at most $2(k-1)$ vertices to $I \cup B$.

Set $\mathcal{I}:=\mathcal{I}-\{v, w\}, \mathcal{S}:=\mathcal{S}-\{v\}$ and $\mathcal{L}:=\mathcal{L}-\{w\}$ and repeat Step 1.1.

Step 1.2: If there exists a vertex $v \in \mathcal{S}$ such that $d(v, w) \leq k$ for a vertex $w \in \mathcal{S}$ with $v \neq w$, we can connect the vertices $v, u_{v}, w$ and $u_{w}$ to one component by adding at most $k-1$ vertices to $I \cup B$.

Set $\mathcal{I}:=\mathcal{I}-\{v, w\}$ and $\mathcal{S}:=\mathcal{S}-\{v, w\}$ and repeat Step 1.2.

Step 1.3: If there exists a vertex $v \in \mathcal{S}$ such that $d(v, w) \leq k$ for a vertex $w \in I_{2}-(\mathcal{S} \cup \mathcal{L})$, we can connect the vertices $v$ and $u_{v}$ to $w$ by adding at most $k-1$ vertices to $I \cup B$.

Set $\mathcal{I}:=\mathcal{I}-\{v\}$ and $\mathcal{S}:=\mathcal{S}-\{v\}$ and repeat Step 1.3.

Note that after completing Step 1 the set $\mathcal{S}$ is empty and there are at most $\left|I_{1}\right|+2\left|I_{2}\right|-3\left(r_{1}+r_{2}\right)-2 r_{3}$ components left, where $r_{i}$ denotes the number of times Step 1.i was repeated for $i=1,2,3$. Furthermore, we have added at most $(k-1)\left(2 r_{1}+r_{2}+r_{3}\right)$ vertices to $I \cup B$.

Step 2: We consider the vertices in $L_{1}$.

If there exists a vertex $v \in L_{1} \cap \mathcal{L}$, let $w \in I_{1}$ be a $k$-neighbor of $x_{v}$. We can connect the vertices $v, u_{v}$ and $w$ to one component by adding at most $2(k-1)$ vertices to $I \cup B$.

Set $\mathcal{I}:=\mathcal{I}-\{v\}$ and $\mathcal{L}:=\mathcal{L}-\{v\}$ and repeat Step 2 .

Note that after completing Step 2 we have $\mathcal{L} \subseteq L_{2}$ and there are at most $\left|I_{1}\right|+$ $2\left|I_{2}\right|-3\left(r_{1}+r_{2}\right)-2 r_{3}-2 s$ components left, where $s$ denotes the number of times Step 2 was repeated and the numbers $r_{i}$ are defined as above. Furthermore, we have added at most $(k-1)\left(2 r_{1}+r_{2}+r_{3}+2 s\right)$ vertices to $I \cup B$.

Step 3: We consider the vertices in $L_{2}$. Recall that for each vertex $v \in L_{2}$ the vertex $x_{v}$ has a $k$-neighbor $w \in I_{2}$ besides $v$. 
Let $v$ be a vertex in $L_{2} \cap \mathcal{L}$ such that $x_{v}$ has a $k$-neighbor $w \in I_{2}-\mathcal{I}$. We can connect the vertices $v, u_{v}$ and $w$ by adding at most $2(k-1)$ vertices to $I \cup B$. Set $\mathcal{I}:=\mathcal{I}-\{v\}$ and $\mathcal{L}:=\mathcal{L}-\{v\}$ and repeat Step 3 .

Note that after completing Step 3 the sets $\mathcal{I}$ and $\mathcal{L}$ are empty and there are at most $\left|I_{1}\right|+2\left|I_{2}\right|-3\left(r_{1}+r_{2}\right)-2 r_{3}-2 s-2 t$ components left, where $t$ denotes the number of times Step 3 was repeated and the numbers $r_{i}$ and $s$ are defined as above. Furthermore, we have added at most $(k-1)\left(2 r_{1}+r_{2}+r_{3}+2 s+2 t\right)$ vertices to $I \cup B$.

Step 4: We connect the remaining components to one component.

Let $D^{\prime}$ be the set of vertices that consists of $I \cup B$ and all vertices added in Steps 1 to 3 . Since $D^{\prime}$ is a $k$-dominating set of $G$, the remaining at most $\left|I_{1}\right|+2\left|I_{2}\right|-3\left(r_{1}+r_{2}\right)-2 r_{3}-2 s-2 t$ components can be connected to one component by adding at most $\left(\left|I_{1}\right|+2\left|I_{2}\right|-3\left(r_{1}+r_{2}\right)-2 r_{3}-2 s-2 t-1\right) 2 k$ vertices to $D^{\prime}$.

After completing Step 4 we have constructed a connected $k$-dominating set $D \supseteq I \cup B$ by adding at most

$$
(k-1)\left(2 r_{1}+r_{2}+r_{3}+2 s+2 t\right)+\left(\left|I_{1}\right|+2\left|I_{2}\right|-3\left(r_{1}+r_{2}\right)-2 r_{3}-2 s-2 t-1\right) 2 k
$$

vertices to $I \cup B$.

We shall show now that the number of vertices we have have added is less or equal than $(2 k-1)\left|I_{2}\right|+2 k\left(\left|I_{1}\right|-1\right)$. Note that $\left|I_{2}\right|=2 r_{1}+2 r_{2}+r_{3}+s+t$. Then

$$
\begin{aligned}
(k- & 1)\left(2 r_{1}+r_{2}+r_{3}+2 s+2 t\right)+\left(\left|I_{1}\right|+2\left|I_{2}\right|-3\left(r_{1}+r_{2}\right)-2 r_{3}-2 s-2 t-1\right) 2 k \\
& -(2 k-1)\left|I_{2}\right|-2 k\left(\left|I_{1}\right|-1\right) \\
= & (2 k+1)\left|I_{2}\right|-3 k\left(2 r_{1}+2 r_{2}+r_{3}+s+t\right)-k\left(r_{3}+s+t\right) \\
& \quad+(k-1)\left(2 r_{1}+r_{2}+r_{3}+2 s+2 t\right) \\
= & -(k-1)\left(2 r_{1}+2 r_{2}+r_{3}+s+t\right)-k\left(r_{3}+s+t\right)+(k-1)\left(2 r_{1}+r_{2}+r_{3}+2 s+2 t\right) \\
= & -(k-1) r_{2}-k r_{3}-s-t \\
\leq & 0 .
\end{aligned}
$$

If we choose $|I|$ such that $|I|=i r_{k}(T)$, it follows that

$$
\begin{aligned}
\gamma_{k}^{c}(T) & \leq|D| \leq\left|I_{1}\right|+2\left|I_{2}\right|+2 k\left|I_{1}\right|+(2 k-1)\left|I_{2}\right|-2 k \\
& =(2 k+1)|I|-2 k \\
& =(2 k+1) i r_{k}(T)-2 k
\end{aligned}
$$

which completes the proof of this theorem.

As an immediate consequence we get the following corollary. 
Corollary 2.8. If $T$ is a tree with $n_{1}$ leaves, then

$$
i r_{k}(G) \geq \frac{|V(T)|-k n_{1}+2 k}{2 k+1} .
$$

Proof. Since $\gamma_{k}^{c}(T) \geq|V(T)|-k n_{1}$ for each tree $T$, the result follows directly from Theorem 2.7.

Note that, since $\gamma_{k}(G) \geq i r_{k}(G)$ for each graph $G$, Corollary 2.8 is also a generalization of Corollary 2.3. The following theorem provides a class of examples that shows that the bound presented in Theorem 2.7 is sharp.

Theorem 2.9 (Meierling \& Volkmann [9] 2005; Cyman, Lemanska \& Raczek [2] 2006). Let $\mathcal{R}$ denote the family of trees in which the distance between each pair of distinct leaves is congruent $2 k$ modulo $(2 k+1)$. If $T$ is a tree with $n_{1}$ leaves, then

$$
\gamma_{k}(T)=\frac{|V(T)|-k n_{1}+2 k}{2 k+1}
$$

if and only if $T$ belongs to the family $\mathcal{R}$.

Remark 2.10. The graph in Figure 1 shows that the construction presented in the proof of Theorem 2.7 does not work if we allow the graph to contain cycles. It is easy to see that $I=\left\{v_{1}, v_{2}\right\}$ is an ir $r_{2}$-set of $G$ and that $D=\left\{u_{1}, u_{2}, x_{1}, x_{2}, x_{3}\right\}$ is a $\gamma_{2}^{c}$-set of $G$. Following the construction in the proof of Theorem 2.7, we have $I_{1}=\emptyset, I_{2}=\left\{v_{1}, v_{2}\right\}$ and $B=\left\{u_{1}, u_{2}\right\}$ and consequently, $D^{\prime}=I_{2} \cup B \cup\left\{x_{1}, x_{2}, x_{3}\right\}$. But $\left|D^{\prime}\right|=7 \not \leq 6=$ $(2 \cdot 2+1)|I|-2 \cdot 2$ and $D$ contains none of the vertices of $I$.

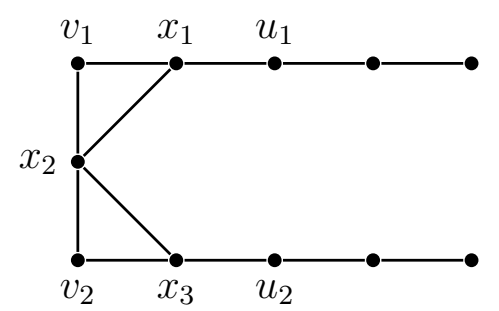

Figure 1.

Nevertheless, we think that the following conjecture is valid.

Conjecture 2.11. If $G$ is a connected graph, then

$$
\gamma_{k}^{c}(G) \leq(2 k+1) i r_{k}(G)-2 k .
$$

Now we analyze the relation between the connected distance domination number and the total distance domination number of a graph. 
Theorem 2.12. If $G$ is a connected graph, then

$$
\gamma_{k}^{c}(G) \leq \frac{3 k+1}{2} \gamma_{k}^{t}(G)-2 k
$$

Proof. Let $G$ be a connected graph and let $D$ be a total $k$-dominating set of $G$ of size $\gamma_{k}^{t}(G)$. Each vertex $x \in D$ is in distance at most $k$ of a vertex $y \in D-\{x\}$. Thus we get a dominating set of $G$ with at most $\lfloor|D| / 2\rfloor$ components by adding at most $\lceil|D| / 2\rceil(k-1)$ vertices to $D$. As in the proof of Lemma 2.4, the resulting components can be joined to a connected $k$-dominating set $\left|D^{\prime}\right|$ by adding at most $(\lfloor|D| / 2\rfloor-1) 2 k$ vertices. Consequently,

$\gamma_{k}^{c}(G) \leq\left|D^{\prime}\right| \leq|D|+\left\lceil\frac{|D|}{2}\right\rceil(k-1)+\left(\left\lfloor\frac{|D|}{2}\right\rfloor-1\right) 2 k \leq \frac{3 k+1}{2}|D|-2 k=\frac{3 k+1}{2} \gamma_{k}^{t}(G)-2 k$

and the proof is complete.

For distance $k=1$ we obtain the following result.

Corollary 2.13 (Favaron \& Kratsch [4] 1991). If $G$ is a connected graph, then

$$
\gamma^{c}(G) \leq 2 \gamma^{t}(G)-2
$$

The following example shows that the bound presented in Theorem 2.12 is sharp.

Example 2.14. Let $P$ be the path on $n=(3 k+1) r$ vertices with $r \in \mathbb{N}$. Then $\gamma_{k}^{c}(P)=$ $n-2 k, \gamma_{k}^{t}(P)=2 r$ and thus, $\gamma_{k}^{c}(P)=\frac{3 k+1}{2} \gamma_{k}^{t}(P)-2 k$.

\section{References}

[1] E.J. Cockayne, S.T. Hedetniemi and D.J. Miller: Properties of hereditary hypergraphs and middle graphs, Canad. Math. Bull. 21 (1978), 461-468.

[2] J. Cyman, M. Lemańska and J. Raczek: Lower bound on the distance $k$-domination number of a tree, Math. Slovaca 56 (2006), no. 2, 235-243.

[3] P. Duchet, H. Meyniel: On Hadwiger's number and the stability number, Ann. Discrete Math. 13 (1982), 71-74.

[4] O. Favaron and D. Kratsch: Ratios of domination parameters, Advances in graph theory, Vishwa, Gulbarga (1991), 173-182.

[5] J.H. Hattingh and M.A. Henning: Distance irredundance in graphs, Graph Theory, Combinatorics, and Applications, John Wiley \& Sons, Inc. 1 (1995) 529-542.

[6] T.W. Haynes, S.T. Hedetniemi and P.J. Slater: Fundamentals of Domination in Graphs, Marcel Dekker, New York (1998).

[7] T.W. Haynes, S.T. Hedetniemi and P.J. Slater: Domination in Graphs: Advanced Topics, Marcel Dekker, New York (1998). 
[8] M.A. Henning, O.R. Oellermann and H.C. Swart: Bounds on distance domination parameters, J. Combin. Inform. System Sci. 16 (1991) 11-18.

[9] D. Meierling and L. Volkmann: A lower bound for the distance $k$-domination number of trees, Result. Math. 47 (2005), 335-339.

[10] A. Meir and J.W. Moon: Relations between packing and covering number of a tree, Pacific J. Math. 61 (1975), 225-233.

[11] J. Topp and L. Volkmann: On packing and covering numbers of graphs, Discrete Math. 96 (1991), 229-238. 